\title{
Dynamic Phase Transition in a Time-Dependent Ginzburg-Landau Model in an Oscillating Field
}

\author{
H. Fujisaka, ${ }^{1, *}$ H. Tutu, ${ }^{1, \dagger}$ and P. A. Rikvold ${ }^{2, \dagger}$ \\ ${ }^{1}$ Department of Applied Analysis and Complex Dynamical Systems, \\ Graduate School of Informatics, \\ Kyoto University, Kyoto 606-8501, Japan \\ ${ }^{2}$ Center for Materials Research and Technology, \\ School of Computational Science and Information Technology, \\ and Department of Physics, \\ Florida State University, Tallahassee, Florida 32306-4350, USA
}

(May 30, 2018)

\begin{abstract}
The Ginzburg-Landau model below its critical temperature in a temporally oscillating external field is studied both theoretically and numerically. As the frequency or the amplitude of the external force is changed, a nonequilibrium phase transition is observed. This transition separates spatially uniform, symmetry-restoring oscillations from symmetry-breaking oscillations. Near the transition a perturbation theory is developed, and a switching phenomenon is found in the symmetry-broken phase. Our results confirm the equivalence of the present transition to that found in Monte Carlo simulations of kinetic Ising systems in oscillating fields, demonstrating that the nonequilibrium phase transition in both cases belongs to the universality class of the equilibrium Ising model in zero field. This conclusion is in agreement with symmetry arguments [G. Grinstein, C. Jayaprakash, and Y. He, Phys. Rev. Lett. 55, 2527 (1985)] and recent numerical results [G. Korniss, C. J. White, P. A. Rikvold, and M. A. Novotny, Phys. Rev. E (submitted)]. Furthermore, a theoretical result for the structure function of the local magnetization with thermal noise, based on the Ornstein-Zernike approximation, agrees well with numerical results in one dimension.
\end{abstract}

PACS: 64.60.Ht, 05.45.-a, 05.10.Gg, 05.50.+q

\section{INTRODUCTION}

Bistable systems that are driven between their two states by a periodically oscillating external force are common,

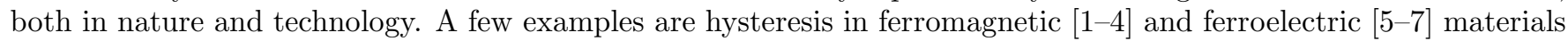
driven by oscillating applied fields, electrochemical adsorbate systems driven across a phase transition by an oscillating electrode potential [8 [10], and liquid crystals driven through a phase transition by pressure oscillations [11]. In this paper we use magnetic language, henceforth referring to the order parameter as the magnetization and the oscillating force as the magnetic field.

When the field oscillates at a sufficiently low frequency, the driven system essentially follows the field, switching between its two states in a symmetry-restoring oscillation (SRO) with the same period, provided that the amplitude of the external force is larger than a critical value which depends on the temperature and the system's spatial dimension. At high driving frequencies, on the other hand, the system is unable to follow the field and instead settles down to a symmetry-breaking oscillation (SBO) around one or the other of its zero-field stable states.

Over the last decade it has become evident that the boundary between the SRO and SBO regimes corresponds to a singularity that appears to have all the hallmarks of a genuine second-order phase transition. It is therefore appropriate to consider the SRO and SBO as dynamic phases of this far-from-equilibrium system. Characteristic features of this nonequilibrium phase transition include a power-law dependence of the amplitude of the SBO on the amount by which the frequency $\Omega$ exceeds its field and temperature dependent critical value $\Omega_{c}$, as well as critical slowing-down 12]. The probability density of the period-averaged magnetization exhibits a one-peak structure for $\Omega<\Omega_{c}$ and a two-peak structure for $\Omega>\Omega_{c}$ [13 17]. In spatially extended bistable systems, such as the twodimensional kinetic Ising model below its critical temperature, the transition also displays a divergent correlation length and finite-size scaling properties analogous to those familiar from equilibrium phase transitions [13 17]. It has become common to refer to this symmetry-breaking transition as the "dynamic phase transition" (DPT).

The DPT was first observed in numerical solutions of a deterministic mean-field equation of motion for a ferromagnet in an oscillating field [18,19, and it has subsequently been seen and studied in numerous Monte Carlo (MC) simulations of kinetic Ising systems 12 17,20 25], as well as in further mean-field studies [12,21,23,26,27. It may also have been observed experimentally in ultrathin Co films on $\mathrm{Cu}(100)$ [3, 1 . Reviews of earlier research on the DPT and related phenomena are found in Refs. 28,29]. 
Finite-size scaling analysis of MC data for the DPT in the two-dimensional kinetic Ising model at sub-critical temperatures provides strong numerical evidence that this nonequilibrium critical phenomenon belongs to the same universality class as the equilibrium phase transition in the two-dimensional Ising model in zero field [13 16]. While this result may seem surprising at first, it is consistent with a symmetry argument by Grinstein, Jayaprakash, and He [30]. This argument states that continuous ordering transitions of fully probabilistic cellular automata with Ising-like "up-down" symmetry (of which the kinetic Ising model in an oscillating field is an example) should fall in the same universality class as the corresponding Ising model in equilibrium. This implies that such a cellular automaton should possess an underlying coarse-grained effective Hamiltonian at sufficiently large length scales, which determines its universality class.

The purpose of the present paper is to elucidate the origin of the DPT and to clarify the statistical characteristics of the dynamics near the DPT, subject to thermal noise. To this effect we consider a time-dependent Ginzburg-Landau model with thermal noise. The equation of motion for the noise-free version of this model is

$$
\dot{\psi}(\mathbf{r}, t)=\psi-\psi^{3}+\nabla^{2} \psi+h \cos (\Omega t),
$$

where $\psi(\mathbf{r}, t)$ is the continuous scalar magnetization field, and $h$ and $\Omega$ are the amplitude and frequency of the spatially uniform external magnetic field, respectively. In zero applied field, Eq. (1) is identical to the conventional GinzburgLandau equation for the Ising model below its critical temperature. The effects of thermal noise on the system are expressed by the stochastic differential equation

$$
\dot{\psi}(\mathbf{r}, t)=\psi-\psi^{3}+\nabla^{2} \psi+h \cos (\Omega t)+R(\mathbf{r}, t),
$$

where $R(\mathbf{r}, t)$ is a Gaussian white thermal noise. In this paper we show that Eqs. (1) and (2) give rise to a bifurcation line in the $(\Omega, h)$ plane. Near this bifurcation line the equations yield an effective Hamiltonian for a dynamic order parameter. This effective Hamiltonian is in the same universality class as the equilibrium Ising model in zero field, and its existence provides explicit confirmation of the symmetry argument of Ref. [30] for this far-from-equilibrium system.

Equations (11) and (2) with $h=0$ give rise to two degenerate ordered solutions only for systems of spatial dimension $d \geq 2$ at temperatures below criticality. These conditions will be assumed hereafter, unless otherwise is explicitly stated.

The present paper is organized as follows. In Sec. II we show that the spatially uniform oscillation of Eq. (1) undergoes a bifurcation as $\Omega$ is increased, which separates the symmetry-restoring and symmetry-breaking dynamic phases. In Sec. III we develop a Landau expansion near the bifurcation, which is used to explain the switching phenomenon observed in a system subject to thermal noise. In Sec. IV we show that theoretical results for spatial power spectra of spin fluctuations (structure functions) obtained by the Landau expansion are in good agreement with numerical experiments for a one-dimensional system. A summary and conclusions are given in Sec. $\square$.

\section{BIFURCATION OF THE SYMMETRY-RESTORING OSCILLATION}

In this Section we concentrate on the uniform solutions of the the noise-free system described by Eq. (1). The effects of spatial fluctuations and thermal noise will be discussed in Sec. III.

It is easy to see that Eq. (11) has a spatially uniform oscillation,

$$
\dot{\psi}(t)=\psi-\psi^{3}+h \cos (\Omega t)
$$

provided that spatially periodic boundary conditions are used. Without loss of generality, $h$ and $\Omega$ are taken as positive. Eventually, $\psi(t)$ always exhibits a periodic oscillation of frequency $\Omega$ for any choice of $h, \Omega$, and the initial value $\psi(0)$. It exhibits no other periodic or chaotic oscillations. This is so because the dynamical system (3) is dissipative and has only two degrees of freedom.

One should be careful when discussing the dynamics near $\Omega=0$. By shifting time as $t \rightarrow t-\pi /(2 \Omega)$, Eq. (3) reduces to

$$
\dot{\psi}(t)=\psi-\psi^{3}+h \sin (\Omega t) .
$$

If one puts $\Omega=0$ in Eqs. (3) and (㺼) while keeping $h$ finite, they have different fixed points. The period $T(\equiv 2 \pi / \Omega)$ of the applied field tends to infinity as $\Omega \rightarrow 0$. One should therefore discuss the long-time behavior of $\psi(t \gg T)$ at finite $\Omega$, and then take the limit $\Omega \rightarrow 0$. The above discrepancy originates from the interchange of the limits $t \rightarrow \infty$ and $\Omega \rightarrow 0$. If one takes the limits correctly, the long-time behaviors of Eqs. (3) and (4) give the same results. 
For $h=0, \psi(t)$ eventually approaches one of the stable fixed points, $\psi_{0}= \pm 1$. Then, under an applied field $h \cos (\Omega t)$ with a small amplitude, it is easy to see that $\psi(t)$ exhibits a periodic oscillation. In fact, to first order in $h$, Eq. (3) is solved by

$$
\psi(t)= \pm 1+\frac{h}{4+\Omega^{2}}[2 \cos (\Omega t)+\Omega \sin (\Omega t)]
$$

for $t \rightarrow \infty$. This is a SBO since $\psi(t)$ oscillates near either $\psi=+1$ or -1 , depending on the initial condition. We thus expect that Eq. (3) exhibits a symmetry-breaking periodic oscillation in the regime of relatively weak $h$.

Let $\psi(t)$ be a solution of Eq. (3). It is then easy to show that $\hat{\psi}(t)$ given by

$$
\hat{\psi}(t)=-\psi\left(t+\frac{T}{2}\right)
$$

is also always a solution of Eq. (3), including even the transient process. As a special case (see Eq. (27) below), Eq. (3) has a particular solution with the symmetry

$$
\psi(t)=-\psi\left(t+\frac{T}{2}\right) .
$$

If the dynamical behavior satisfies the symmetry (7), one obtains

$$
\int_{0}^{T} \psi(t) e^{i \ell \Omega t} d t=0,(\ell=0, \pm 2, \pm 4, \cdots) .
$$

However, the fact that the system (3) has the symmetry (7) does not necessarily mean that the dynamical behavior always exhibits this symmetry. In fact, as discussed above for small $h$ and as shown below, Eq. (3) has a stable symmetry-breaking solution for a certain range of $h$ and $\Omega$.

As discussed above, the dynamics in a weak external field shows a SBO. This implies that the SRO, if it exists, should do so for a relatively large amplitude of the external field. This also suggests that there should exist a transition between the SBO and the SRO, provided that the SRO stably exists. This is an immediate consequence of the symmetry consideration.

For the moment, let us consider the parameter values $h=1.0$, and $\Omega=1.08$ and 1.1. For these parameter values, the system has attractors as shown in Fig. 1. Throughout this paper, the numerical integration of Eq. (3) is carried out by using the fourth-order Runge-Kutta algorithm with the time increment $\Delta t=T / 1024$ for all frequencies. The attractors are limit cycles of period $T$. They have the symmetry $(7)$ for $\Omega=1.08$, but are asymmetric for $\Omega=1.1$. The above considerations suggest the existence of a phase transition between these different characteristic oscillations. Figure 2 shows the hysteresis loops, i.e., the dependence of $\psi(t)$ on $h(t) \equiv h \cos (\Omega t)$ for $\Omega$ below and above $\Omega_{c}$, the critical frequency separating the interchange of the symmetric and non-symmetric oscillations. Numerically, we find $\Omega_{c} \approx 1.095$ for $h=1.0$.

Next we consider the stability of the attractor with the symmetry (7), shown in Fig. 11, as $\Omega$ is increased at fixed $h$. The stability of a periodic oscillation is discussed as follows. Let $\psi(t)$ be a particular solution of Eq. (3) on an attractor, which may be either stable or unstable. In order to examine its linear stability, we seek the temporal evolution of the deviation $\delta \psi(t)$ from this solution. Then, $\delta \psi(t)$ obeys the equation of motion,

$$
\dot{\delta \dot{\psi}}(t)=\left[1-3(\psi(t))^{2}\right] \delta \psi(t)
$$

Since $\psi(t)$ is periodic with period $T, \delta \psi(t)$ is solved as

$$
\delta \psi(t)=B(t) e^{\Lambda t} \delta \psi(0) .
$$

If we define

$$
\Lambda=1-3 \overline{\psi^{2}}=1-3 \frac{1}{T} \int_{0}^{T}(\psi(s))^{2} d s
$$

then

$$
B(t)=\exp \left[-3 \int_{0}^{t}\left\{\left(\psi\left(t^{\prime}\right)\right)^{2}-\overline{\psi^{2}}\right\} d t^{\prime}\right]
$$


is a periodic function of period $T$, i.e., $B(t+T)=B(t)$. Here we have defined the period-average of $f(t)$ as $\overline{f(t)}=T^{-1} \int_{0}^{T} f(t+s) d s$. The results (10-12) follow from the Floquet theorem [31]. The quantity $\Lambda$ is called the Floquet exponent and indicates the stability of the periodic oscillation under consideration, i.e., $\psi(t)$ is linearly stable (unstable) if $\Lambda<0(>0)$. Numerical results for $\Lambda$ calculated by Eq. (11) are shown in Fig. 3. For $\Omega$ below $\Omega_{c}$, the critical value for a given $h, \Lambda$ takes a negative value, which is denoted by $\lambda$. The limit cycle for $\Omega<\Omega_{c}$ is symmetric as shown in Figs. 1) (a) and 2(a). As $\Omega$ is gradually increased, the Floquet exponent approaches zero and again takes a negative value for $\Omega>\Omega_{c}$. Figures 1 (b) and 2 (b) show the stable attractors (solid curves) corresponding to the limit cycles for $\Omega>\Omega_{c}$. Figure shows the stability regions of the SRO and the SBO. In the SBO region, one finds that there exist two attractors, $\mathrm{C}_{-}$and $\mathrm{C}_{+}$, one of which is chosen depending on the initial condition. For $\Omega>\Omega_{c}$, there is also a symmetry-restoring unstable limit cycle, whose Floquet exponent is denoted by $\lambda_{u}$ in Fig. 3, and whose trajectory is depicted by the dashed curves in Figs. 1(b) and 2(b). The transition at $\Omega_{c}$ is continuous, as is expected from the frequency dependence of the Floquet exponent shown in Fig. 3 .

The unstable limit cycle, i.e., the SRO for $\Omega>\Omega_{c}$, is numerically obtained as follows. Taking an initial value $\psi_{n}$ at time $t_{n}=n T$, then integrating Eq. (3) until $t_{n+1}=t_{n}+T$, we obtain $\psi_{n+1}$. In this way we get the $\psi_{n+1}$ vs $\psi_{n}$ curve,

$$
\psi_{n+1}=g\left(\psi_{n}\right) .
$$

Examples of numerically obtained $g(\psi)$ are shown in Fig. F. Figure (a) is for $\Omega<\Omega_{c}$, and Fig. 司(b) is for $\Omega>\Omega_{c}$. Depending on $\Omega$, there are one or three fixed points $\psi_{f}$ satisfying $\psi_{f}=g\left(\psi_{f}\right)$, which correspond to cross-sections of limit-cycle attractors. The stability of a limit cycle is determined by the slope of $g(\psi)$ at $\psi=\psi_{f}$, i.e., the Floquet exponent is given by

$$
\Lambda=\frac{1}{T} \ln \left|g^{\prime}\left(\psi_{f}\right)\right| .
$$

The unstable periodic orbit shown in Figs. 1(b) and 2(b) is the one numerically integrated with the initial value $\psi_{u}$, the unstable fixed point. The temporal evolutions of one unstable and two stable oscillations are shown in Fig. 6. One should note that if the stroboscopic map is constructed for times $t_{n}=\tau+n T$, the form of $g(\psi)$ depends on $\tau$. However, the number of fixed points of $\psi_{n+1}=g\left(\psi_{n}\right)$ and the corresponding slopes, which yield the Floquet exponents for the fixed points, are independent of $\tau$.

The bifurcation point $\Omega_{c}$ depends on $h$. The theoretical bifurcation curve given by the solid curve in Fig. 1 is determined as follows. We first expand $\psi(t)$ as the Fourier series

$$
\psi(t)=\sum_{\ell=-\infty}^{\infty} \psi_{\ell}(t) e^{i \ell \Omega t},
$$

where $\psi_{-\ell}=\psi_{\ell}^{*}$. The temporal evolution of the coefficients $\left\{\psi_{\ell}(t)\right\}$ is assumed to be much slower than the time scale T. Inserting Eq. (15) into Eq. (3) and comparing the coefficients on both sides of the equation, we obtain

$$
\dot{\psi}_{\ell}+i \ell \Omega \psi_{\ell}=\psi_{\ell}-\sum_{m} \sum_{n} \psi_{m} \psi_{n} \psi_{\ell-m-n}+\frac{1}{2}\left(\delta_{\ell, 1}+\delta_{\ell,-1}\right) h .
$$

From the symmetry argument, Eq. (3) may have a solution with the symmetry (7). If the limit cycle under consideration is symmetric, we find from Eq. (8) that

$$
\psi_{\ell}=0 \quad \text { for } \ell=0, \pm 2, \pm 4, \cdots .
$$

We now consider the stability of this symmetric oscillation. As the simplest non-trivial approximation, we use the truncation $\ell=0$ and \pm 1 , which yields

$$
\begin{gathered}
\dot{\psi}_{0}=\left[1-6\left|\psi_{1}\right|^{2}-\psi_{0}^{2}\right] \psi_{0}, \\
\dot{\psi}_{1}+i \Omega \psi_{1}=\left[1-3\left|\psi_{1}\right|^{2}-3 \psi_{0}^{2}\right] \psi_{1}+\frac{1}{2} h .
\end{gathered}
$$

The above equations have a SRO $\left(\psi_{0}=0\right)$ provided that $1-6\left|\psi_{1}\right|^{2}<0$. On the other hand, for $1-6\left|\psi_{1}\right|^{2}>0$ the steady-state value of $\psi_{0}$ does not vanish, which implies the emergence of a SBO. Therefore, we find that the 
boundary between the regions of stability of the SRO and SBO is determined by $\left|\psi_{1}^{\mathrm{ss}}\right|^{2}=\frac{1}{6}$. Combining this with the steady-state value of $\psi_{1}^{\text {ss }}$ obtained from Eq. (19), the bifurcation point $\Omega_{c}$ for fixed $h$ is determined by

$$
\Omega_{c}=\sqrt{\frac{3}{2}\left(h^{2}-\frac{1}{6}\right)}
$$

or, equivalently,

$$
h=\sqrt{\frac{2}{3}\left(\frac{1}{4}+\Omega_{c}^{2}\right)} .
$$

One finds that this kind of bifurcation is observed for $h$ larger than a critical value, $1 / \sqrt{6}$ in the above approximation. The curve given by Eq. (21) corresponds to the transition line, which in Fig. 4 is compared with results from numerical integration of Eq. (3). For $\Omega<\Omega_{c}$, there is only one type of periodic motion, namely the symmetry-restoring one. For $\Omega>\Omega_{c}$, on the other hand, there are two types of oscillations: one is the unstable SRO, and the other is represented by the two stable SBOs, one of which is observed for a given initial condition.

Here a comment on the critical value of $h$ under a static field, $\Omega=0$, should be added. The above approximation yields the critical value $1 / \sqrt{6} \approx 0.408$. On the other hand, the standard calculation in mean-field theory yields the spinodal field as the field where the metastable minimum in the $\psi^{4}$ potential disappears. This condition requires that the equations,

$$
\begin{aligned}
\dot{\psi} & =\psi-\psi^{3}+h=0 \\
\frac{\partial \dot{\psi}}{\partial \psi} & =1-3 \psi^{2}=0
\end{aligned}
$$

are simultaneously satisfied. The second equation gives $\psi_{\text {spinodal }}= \pm 1 / \sqrt{3}$ which, when inserted into the first equation, yields $h_{\text {spinodal }}=2 \sqrt{3} / 9 \approx 0.385$. This value is about $6 \%$ below that obtained in the above discussion. We carried out numerical calculations for values of $\Omega$ as small as 0.05 . The numerical results seem to be closer to $h_{\text {spinodal }}$ than to the present approximate value, $1 / \sqrt{6}$. However, calculations at even smaller $\Omega$, which were not feasible in the present study, would be needed to reach a firm conclusion. A sharp decrease of the critical value of $h$ as $\Omega$ is decreased may suggest the possibility that the transition curve may have a kind of singularity, i.e., that $d h / d \Omega$ might diverge as $\Omega$ approaches zero 26.

Next we evaluate how the amplitude of the SBO develops for $\Omega$ above $\Omega_{c}$. The steady-state values $\psi_{0}^{\text {ss }}$ and $\psi_{1}^{\text {ss }}$ are obtained by setting $\psi_{0}^{\mathrm{ss}}=\sin \theta$ and $\psi_{1}^{\mathrm{ss}}=6^{-\frac{1}{2}} \cos \theta \cdot e^{i \alpha}$. For $\Omega<\Omega_{c}, \theta=0$, while $\theta$ is small for $\Omega \gtrsim \Omega_{c}$. A short calculation shows that the order parameter $\psi_{0}^{\text {ss }}$ is asymptotically given by

$$
\psi_{0}^{\mathrm{ss}}= \pm c_{1} \sqrt{\Omega-\Omega_{c}}
$$

with $c_{1}=\sqrt{8 \Omega_{c} /\left(4 \Omega_{c}^{2}+11\right)}$. The amplitude and phase of $\psi_{1}^{\text {ss }}$ are given as

$$
\left|\psi_{1}^{s s}\right|=\frac{1}{\sqrt{6}}\left[1-\frac{1}{2} c_{1}^{2}\left(\Omega-\Omega_{c}\right)\right]
$$

and

$$
\alpha=\alpha_{c}+c_{2}\left(\Omega-\Omega_{c}\right)
$$

respectively, where

$$
\begin{aligned}
\cos \alpha_{c} & =\frac{-1}{\sqrt{4 \Omega_{c}^{2}+1}}, \\
\sin \alpha_{c} & =\frac{-2 \Omega_{c}}{\sqrt{4 \Omega_{c}^{2}+1}}, \\
c_{2} & =\frac{22}{4 \Omega_{c}^{2}+11} .
\end{aligned}
$$

The $\Omega$ dependences of the amplitudes of the SRO and SBO are shown in Fig. 7, which was obtained from the stable and unstable fixed points of Eq. (13). 
Numerical integration shows that the time evolutions of the symmetry-breaking orbits $\mathrm{C}_{+}$and $\mathrm{C}_{-}$, which are $\psi_{+}(t)$ and $\psi_{-}(t)$, respectively, are related as

$$
\begin{aligned}
& \psi_{+}(t)=-\psi_{-}\left(t+\frac{T}{2}\right) \\
& \psi_{-}(t)=-\psi_{+}\left(t+\frac{T}{2}\right)
\end{aligned}
$$

(see Fig. 6), where

$$
\dot{\psi}_{ \pm}(t)=\psi_{ \pm}(t)-\left(\psi_{ \pm}(t)\right)^{3}+h \cos (\Omega t) .
$$

The symmetry (27) is just a particular case of the general symmetry relation (6).

We find that the stable limit cycle of Eq. (3) for $\Omega<\Omega_{c}\left(\Omega>\Omega_{c}\right)$ is a symmetry-restoring (symmetry-breaking) oscillation, and that one of the two stable SBOs is chosen, depending on the initial condition. Next, we examine the stability of the uniform (stable) oscillation with negative Floquet exponent against inhomogeneous fluctuations. (Remember that the solution of Eq. (3) is the uniform solution of Eq. (17).) Let $\psi(t)$ be a stable solution of Eq. (3), which implies that its Floquet exponent is negative, i.e., SRO for $\Omega<\Omega_{c}$ or SBO for $\Omega>\Omega_{c}$. The negative Floquet exponent is denoted by $\lambda$ instead of $\Lambda$. Next, let $\tilde{\psi}(\mathbf{r}, t)$ be the deviation from $\psi(t)$, i.e.,

$$
\psi(\mathbf{r}, t)=\psi(t)+\tilde{\psi}(\mathbf{r}, t) .
$$

The Fourier transform of $\tilde{\psi}(\mathbf{r}, t)$ obeys

$$
\dot{\tilde{\psi}}_{\mathbf{k}}(t)=\left[1-3(\psi(t))^{2}\right] \tilde{\psi}_{\mathbf{k}}(t)-k^{2} \tilde{\psi}_{\mathbf{k}}(t),
$$

provided that the deviation is sufficiently small. This equation is solved as

$$
\tilde{\psi}_{\mathbf{k}}(t)=B(t) e^{\lambda_{\mathbf{k}} t} \tilde{\psi}_{\mathbf{k}}(0),
$$

where $B(t)$ is again a periodic function and

$$
\lambda_{\mathbf{k}}=\lambda-k^{2}
$$

is the linear growth rate of the Fourier mode at wave vector $\mathbf{k}$. Since $\lambda<0, \lambda_{\mathbf{k}}$ is always negative, which implies that the uniform oscillation with negative Floquet exponent is linearly stable against inhomogeneous fluctuations with any

wave vector. This implies that the system (1D) eventually approaches a spatially uniform oscillatory motion, provided that there exists no other stable dynamical behavior.

For simplicity, the values of $\Omega_{c}(h)$, obtained above from the spatially uniform solution, will be referred to as the mean-field values of $\Omega_{c}$. In spatially extended systems with thermal noise, the actual values of $\Omega_{c}$ are renormalized by fluctuations.

\section{LANDAU EXPANSION AND THERMAL NOISE EFFECTS}

We now move on to the discussion of the spatially extended system with local interactions, which is described by Eqs. (11) and (2). The noise-free case, Eq. (11), is discussed in Sec. IIIA. The effects of thermal noise, described by Eq. (22), are considered in Sec. IIIB.

\section{A. Landau expansion near the bifurcation point}

Let $\psi_{*}(t)$ be the spatially uniform SRO which obeys Eq. (3) and satisfies the symmetry (7), and let $\Lambda$ be its Floquet exponent, which is calculated by Eq. (11) with $\psi(t)=\psi_{*}(t)$. It is given by $\Lambda=\lambda(<0)$ for $\Omega<\Omega_{c}$, and $\Lambda=\lambda_{u}(>0)$ for $\Omega>\Omega_{c}$, in the notation used in Fig. 3. Expanding $\psi(\mathbf{r}, t)$ around this SRO as

$$
\psi(\mathbf{r}, t)=\psi_{*}(t)+B_{*}(t) \phi(\mathbf{r}, t),
$$

where $B_{*}(t)$ is defined by Eq. (12) with $\psi(t)=\psi_{*}(t)$, and inserting this into Eq. (11), we immediately find 


$$
\dot{\phi}(\mathbf{r}, t)=\left(\Lambda+\nabla^{2}\right) \phi-3 \psi_{*}(t) B_{*}(t) \phi^{2}-\left(B_{*}(t)\right)^{2} \phi^{3} .
$$

Note that because of the particular symmetry (17) in the SRO phase, $B_{*}(t+T / 2)=B_{*}(t)$. Since the coefficients of the above equation are periodic in time, we may use their time-averaged values, noting that the characteristic time $|\Lambda|^{-1}$ of $\phi$ near the transition is much longer than $T$. Making use of the symmetry relation (耳), one can prove that

$$
\frac{1}{T} \int_{0}^{T} \psi_{*}(t) B_{*}(t) d t=\frac{1}{T} \int_{0}^{T} \psi_{*}(t) \exp \left[-3 \int_{0}^{t}\left\{\left(\psi_{*}(s)\right)^{2}-\overline{\psi_{*}^{2}}\right\} d s\right] d t=0 .
$$

Thus, Eq. (34) reduces to

$$
\dot{\phi}(\mathbf{r}, t)=\left(\Lambda+\nabla^{2}\right) \phi-b \phi^{3}=-\tilde{\Gamma} \frac{\delta \mathcal{H}\{\phi\}}{\delta \phi}
$$

with $b=\overline{B_{*}^{2}}$ and

$$
\tilde{\Gamma} \mathcal{H}\{\phi\}=\int\left[-\frac{\Lambda}{2} \phi^{2}+\frac{1}{2}(\nabla \phi)^{2}+\frac{b}{4} \phi^{4}\right] d \mathbf{r} .
$$

Here, $\tilde{\Gamma}$ is a positive constant, which will be determined in an appropriate way below.

\section{B. Switching phenomenon}

The thermal noise effects near the DPT were studied in Refs. 13 [16]. In the present continuum model, the thermal noise $R(\mathbf{r}, t)$ is included in Eq. (22) as a Gaussian white noise satisfying

$$
\begin{aligned}
\langle R(\mathbf{r}, t)\rangle & =0 \\
\left\langle R(\mathbf{r}, t) R\left(\mathbf{r}^{\prime}, t^{\prime}\right)\right\rangle & =2 \Gamma \delta\left(\mathbf{r}-\mathbf{r}^{\prime}\right) \delta\left(t-t^{\prime}\right),
\end{aligned}
$$

where $\langle\cdots\rangle$ denotes the ensemble average. The noise strength $\Gamma$ is proportional to the temperature of the system. In Refs. [13 16] the thermal noise effects were studied by observing the time evolution of the total magnetization in two-dimensional kinetic Ising systems. A switching phenomenon between two asymmetric oscillatory states was observed for values of $\Omega$ slightly above $\Omega_{c}$. The origin of this phenomenon in the present continuous-spin model (2) can be understood as follows. Inserting the expansion (33) into Eq. (2), and approximating the coefficients by their time averages, we obtain

$$
\dot{\phi}(\mathbf{r}, t)=\left(\Lambda+\nabla^{2}\right) \phi-b \phi^{3}+f(\mathbf{r}, t)=-\tilde{\Gamma} \frac{\delta \mathcal{H}\{\phi\}}{\delta \phi(\mathbf{r}, t)}+f(\mathbf{r}, t),
$$

where $f(\mathbf{r}, t)=\left(B_{*}(t)\right)^{-1} R(\mathbf{r}, t)$ is a Gaussian white noise with the strength $\tilde{\Gamma} \equiv \overline{B_{*}^{-2}} \Gamma$. This is also chosen as the value of $\tilde{\Gamma}$ in Eqs. (36) and (37).

Equation (39) is identical to the conventional $\phi^{4}$ Ginzburg-Landau equation in zero external field with a thermal noise term. This equation belongs to the same universality class as the Ising model [32]. This is the mechanism of the DPT and the switching phenomenon observed in Refs. 13 16].

Equation (39) is the central result of this paper, which makes the connection to previous work on the DPT in kinetic Ising models. In those studies, the local dynamic order parameter has been taken as the period-averaged magnetization,

$$
Q_{n}(\mathbf{r})=\overline{\psi\left(\mathbf{r}, t_{n}\right)}=\frac{1}{T} \int_{t_{n}}^{t_{n}+T} \psi(\mathbf{r}, t) d t
$$

where $n=\operatorname{int}(t / T)$. Since $\phi(\mathbf{r}, t)$ depends on $t$ only on time scales much longer than $T$, it can be replaced by a variable $\phi_{n}(\mathbf{r})$. Thus it is easy to show that the traditional form of the local dynamic order parameter is simply proportional to $\phi_{n}(\mathbf{r})$ :

$$
Q_{n}(\mathbf{r})=\frac{1}{T} \int_{t_{n}}^{t_{n}+T} B(s) \phi(\mathbf{r}, s) d s \approx \frac{\bar{B}}{T} \int_{t_{n}}^{t_{n}+T} \phi(\mathbf{r}, s) d s=\bar{B} \phi_{n}(\mathbf{r})
$$


The global dynamic order parameter is simply the spatial average of $Q_{n}(\mathbf{r})$. Thus, any results that are proven for $\phi(\mathbf{r}, t)$ are also proven for the traditional dynamic order parameter, $Q_{n}$.

The Fokker-Planck equation corresponding to Eq. (39) takes the form

$$
\frac{\partial}{\partial t} P\{\phi, t\}=\tilde{\Gamma} \int \frac{\delta}{\delta \phi(\mathbf{r})}\left[P^{*}\{\phi\} \frac{\delta}{\delta \phi(\mathbf{r})}\left(\frac{P\{\phi, t\}}{P^{*}\{\phi\}}\right)\right] d \mathbf{r}
$$

where

$$
P^{*}\{\phi\} \propto e^{-\mathcal{H}^{*}\{\phi\}}
$$

is the steady-state probability density, which has a single (double) peak structure for $\Omega<\Omega_{c}\left(\Omega>\Omega_{c}\right.$ ). Here $\mathcal{H}^{*}\{\phi\}$ is the single- or double-peaked renormalized potential function. For $\Omega \gtrsim \Omega_{c}$, the well separation in $\mathcal{H}^{*}\{\phi\}$ is proportional to $\left(\Omega-\Omega_{c}\right)^{\beta}$, where $\beta$ is the magnetization exponent for the Ising model in the appropriate spatial dimension $[13[16]$.

We postulate that the dynamics of the total magnetization (per unit volume, $V$ ),

$$
\phi_{0}(t) \equiv \frac{1}{V} \int \phi(\mathbf{r}, t) d \mathbf{r}
$$

for Eq. (39), takes approximately two values. This implies that the dynamics can be modeled by the Langevin equation

$$
\dot{\phi}_{0}(t)=\Lambda \phi_{0}(t)-b\left(\phi_{0}(t)\right)^{3}+f_{0}(t)
$$

where $f_{0}(t)$ is a random force with

$$
\begin{aligned}
\left\langle f_{0}(t)\right\rangle & =0 \\
\left\langle f_{0}(t) f_{0}\left(t^{\prime}\right)\right\rangle & =2 \tilde{\Gamma}_{0} \delta\left(t-t^{\prime}\right) .
\end{aligned}
$$

The Fokker-Planck equation is thus approximated by

$$
\frac{\partial}{\partial t} P\left(\phi_{0}, t\right)=\Gamma_{0} \frac{\partial}{\partial \phi_{0}}\left[P^{*}\left(\phi_{0}\right) \frac{\partial}{\partial \phi_{0}}\left(\frac{P\left(\phi_{0}, t\right)}{P^{*}\left(\phi_{0}\right)}\right)\right]
$$

where

$$
P^{*}\left(\phi_{0}\right) \propto e^{-\mathcal{H}_{0}^{*}\left(\phi_{0}\right)},
$$

where $\mathcal{H}_{0}^{*}\left(\phi_{0}\right)$ corresponds to the critical order-parameter distribution for the Ising model [32,34, 35. Except for the absence in the volume-averaged $\phi_{0}$ of spatial variations, this result is analogous to Eq. (43) for $\mathcal{H}^{*}(\phi)$.

As an illustration of the switching behavior, Fig. 8 shows the evolution of the total magnetization obtained by numerically solving Eq. (2) for $d=1$ with $\Omega$ slightly larger than the mean-field value of $\Omega_{c}$. The numerical integration of Eq. (2) was carried out by the second-order stochastic Runge-Kutta (SRK) algorithm [33], dividing the space into lattice points with lattice spacing $\Delta x$ (set to 0.5 throughout this paper) and using

$$
\left(\nabla^{2} \psi\right)_{j}=\frac{\psi_{j-1}-2 \psi_{j}+\psi_{j+1}}{(\Delta x)^{2}}
$$

at the lattice site $j$ with periodic boundary conditions. A switching phenomenon is clearly observed.

The switching phenomenon can be formulated in a different way as follows. The temporal evolutions $\psi_{+}(t)$ and $\psi_{-}(t)$ of the symmetry-breaking orbits, $\mathrm{C}_{+}$and $\mathrm{C}_{-}$, respectively, obey Eq. (28). We study the additive noise effect on the dynamics, adding a weak noise $R$, as in Eq. (2). We also include the spatial variation of the dynamical variable, adding $\nabla^{2} \psi$. Consider a local bistable variable. For $\Omega>\Omega_{c}$, depending on the initial condition, either $\mathrm{C}_{+}$or $\mathrm{C}_{-}$ is selected, provided the noise is absent. If the noise is sufficiently weak, the phase point is almost always on either $\mathrm{C}_{+}$and $\mathrm{C}_{-}$. When $\psi_{+}$and $\psi_{-}$are close, the phase point can switch to the other orbit through the noise effect. The above picture can be mathematically formulated as follows. Let $a(t)$ be a variable which takes the values $+1(-1)$, provided the phase point is on $\mathrm{C}_{+}\left(\mathrm{C}_{-}\right)$at time $t$. Then the temporal evolution of $a(t)$ is approximately described by the switching dynamics. The above picture is generalized by introducing the position dependent variable $a(\mathbf{r}, t)$, defined by

$$
\psi(\mathbf{r}, t)=\frac{1-a(\mathbf{r}, t)}{2} \psi_{+}\left(t+t_{0}^{+}\right)+\frac{1+a(\mathbf{r}, t)}{2} \psi_{-}\left(t+t_{0}^{-}\right)
$$


where $t_{0}^{ \pm}$are certain initial times. The variable $a(\mathbf{r}, t)$ indicates whether the local magnetization $\psi(\mathbf{r}, t)$ is close to $\psi_{+}\left(t+t_{0}^{+}\right)$or $\psi_{-}\left(t+t_{0}^{-}\right)$. Namely, if $a(\mathbf{r}, t)$ is near +1 or $-1, \psi(\mathbf{r}, t)$ is close to $\psi_{+}\left(t+t_{0}^{+}\right)$or $\psi_{-}\left(t+t_{0}^{-}\right)$, respectively. Without loss of generality, $t_{0}^{ \pm}$are chosen such that $\cos \left(\Omega t_{0}^{ \pm}\right)=1$, and therefore we put $t_{0}^{ \pm}=0$. Inserting Eq. (50) into Eq. (2), after some algebra we rigorously get

$$
\dot{a}(\mathbf{r}, t)=\frac{1}{4}\left(1-a^{2}\right)\left[\left(\psi_{+}(t)-\psi_{-}(t)\right)^{2} a-3\left\{\left(\psi_{+}(t)\right)^{2}-\left(\psi_{-}(t)\right)^{2}\right\}\right]+\nabla^{2} a+g(\mathbf{r}, t)
$$

with

$$
g(\mathbf{r}, t)=\frac{-2 R(\mathbf{r}, t)}{\psi_{+}(t)-\psi_{-}(t)} .
$$

Furthermore, as long as the thermal noise is weak, the average switching time between $a=+1$ and -1 is long. The temporally periodic coefficients can therefore be replaced by their average values, which reduces Eq. (51) to

$$
\dot{a}=\mu\left(1-a^{2}\right) a+\nabla^{2} a+g(\mathbf{r}, t)
$$

with

$$
\mu=\frac{1}{4} \overline{\left(\psi_{+}(t)-\psi_{-}(t)\right)^{2}}=\frac{1}{4} \overline{(B(t))^{2}\left(\phi_{+}(t)-\phi_{-}(t)\right)^{2}}(>0),
$$

where $\overline{\psi_{+}^{2}}=\overline{\psi_{-}^{2}}$ by symmetry. The quantities $B(t)$ and $\phi_{ \pm}(t)$ are the same as in Sec. II. Equation (53) again takes the form of the Ginzburg-Landau equation with a double-well potential, with stable fixed points $a= \pm 1$, provided that the spatial variation of $a$ and the noise are neglected. Equation (53) shows the switching phenomenon.

\section{STRUCTURE FUNCTION IN THE SRO PHASE}

In this Section, we study the structure function in the SRO phase $(\lambda<0)$ in a one-dimensional system. We define the structure function,

$$
I_{\mathbf{k}}=\left\langle\left\langle\left.\overline{\psi_{\mathbf{k}}(t)}\right|^{2}\right\rangle,\right.
$$

for the Fourier transform $\overline{\psi_{\mathbf{k}}(t)}$ of the period-averaged order parameter,

$$
\overline{\psi(\mathbf{r}, t)} \equiv \frac{1}{T} \int_{t}^{t+T} \psi(\mathbf{r}, s) d s .
$$

We obtain $I_{\mathbf{k}}$ by numerically solving Eq. (2) in a one-dimensional system for several values of $\Omega$ in the SRO phase as shown in Fig. 9. One characteristic feature of the numerically obtained structure function is the $k^{-4}$ behavior observed for relatively large $k$. Here, $I_{\mathbf{k}}$ can be evaluated using the Landau expansion with thermal noise as follows. By inserting Eq. (33) into Eq. (56) and using the approximation [see also Eqs. (40) and (41)]

$$
\overline{\psi(\mathbf{r}, t)}=\frac{1}{T} \int_{t}^{t+T} B(s) \phi(\mathbf{r}, s) d s \approx \frac{\bar{B}}{T} \int_{t}^{t+T} \phi(\mathbf{r}, s) d s,
$$

the structure function is evaluated as

$$
I_{\mathbf{k}}=\frac{\bar{B}^{2}}{T^{2}} \int_{0}^{T} d s \int_{0}^{T} d s^{\prime}\left\langle\phi_{\mathbf{k}}(s) \phi_{\mathbf{k}}^{*}\left(s^{\prime}\right)\right\rangle .
$$

Using the linearized form of the equation of motion for $\phi(\mathbf{r}, t)$, Eq. (39), which yields

$$
\dot{\phi}(\mathbf{r}, t)=\left(\lambda+\nabla^{2}\right) \phi(\mathbf{r}, t)+f(\mathbf{r}, t),
$$

we immediately get the correlation function

$$
\left\langle\phi_{\mathbf{k}}(s) \phi_{\mathbf{k}}^{*}\left(s^{\prime}\right)\right\rangle=\frac{\tilde{\Gamma}}{\gamma_{\mathbf{k}}} e^{-\gamma_{\mathbf{k}}\left|s-s^{\prime}\right|},
$$


where

$$
\gamma_{\mathbf{k}}=|\lambda|+k^{2}
$$

is the damping rate for the fluctuation $\phi_{\mathbf{k}}(t)$. Substitution of Eq. 600 into Eq. (58) yields

$$
I_{\mathbf{k}}=p \Gamma T G\left(\gamma_{\mathbf{k}} T\right),
$$

where $p=\bar{B}^{2} \overline{B^{-2}}$ and

$$
G(x)=\frac{2}{x^{2}}\left(1-\frac{1-e^{-x}}{x}\right)
$$

is a scaling function. We thus find that the structure function $I_{\mathbf{k}}$ behaves asymptotically as $I_{\mathbf{k}} \sim \gamma_{\mathbf{k}}^{-1}$ for $\gamma_{\mathbf{k}} T \ll 1$ and as $\sim \gamma_{\mathbf{k}}^{-2} T^{-1}$ for $\gamma_{\mathbf{k}} T \gg 1$. In the former case, the relation $I_{k} \propto \gamma_{k}^{-1}$ is identical to the Ornstein-Uhlenbeck form which holds for $T \rightarrow 0$. The latter characteristic explains the $k^{-4}$ behavior in the large- $k$ regime. This unexpected behavior originates from the temporal averaging procedure (56), which ensures that the interfaces between regions of positive and negative values of $\overline{\psi_{\mathbf{k}}(t)}$ are not thin. As a result, $I_{\mathbf{k}}$ does not obey "Porod's law," $I_{\mathrm{k}} \sim k^{-(d+1)}$ [36], which would yield $I_{\mathrm{k}} \sim k^{-2}$ for the present case of $d=1$.

The solid curves in Fig. 9 correspond to the theoretical result (62-63). Although this linear model for the fluctuations agrees quite well with the numerical simulations, particularly in the large-wavenumber regime and for strong thermal noise, nonlinear fluctuations play a significant role and the linear model eventually breaks down.

\section{SUMMARY AND CONCLUSION}

In this paper we used a time-dependent Ginzburg Landau model in a temporally oscillating external field to understand the dynamic phase transition (DPT) observed in Monte Carlo simulations of the corresponding kinetic Ising model below its critical temperature $12,17,21,24,28,29]$. Analyzing the stability of spatially uniform oscillations, we found a bifurcation of the symmetry-restoring oscillation (SRO), which leads to the onset of a symmetry-breaking oscillation (SBO). Developing a Landau expansion near the bifurcation point, including additive thermal noise, we obtained an effective Ginzburg Landau Hamiltonian for the amplitude of the SBO, which is proportional to the dynamic order parameter which characterizes the DPT. This effective Hamiltonian has the same form as the standard $\phi^{4}$ Ginzburg Landau Hamiltonian in zero external field, which describes the long-range properties of the Ising model in the critical region [32]. This result implies that the DPT belongs to the same universality class as the equilibrium Ising model in zero external field, in agreement with recent high-precision numerical results from Monte Carlo simulations [13 16], as well as with a symmetry argument which states that the equilibrium Ising universality class should encompass all stochastic cellular automata with Ising "up-down" symmetry [30].

To the best of our knowledge, the work presented here is the first in which an effective Hamiltonian has been explicitly derived for a far-from equilibrium phase transition, confirming that the transition belongs to the same universality class as the equilibrium Ising model in zero field. The result represents a significant expansion of the realm of validity of symmetry arguments from equilibrium to nonequilibrium phase transitions.

\section{ACKNOWLEDGMENTS}

We appreciate useful comments on the manuscript by G. Brown.

P. A. R. acknowledges the hospitality in 1998 of the Department of Fundamental Sciences, Faculty of Integrated Human Studies, Kyoto University, which enabled the authors to begin their collaboration.

This study was partially supported by Grant-in-Aid for Scientific Research No. 11837009 from the Ministry of Education, Science, Sports and Culture of Japan. Work at Florida State University was supported in part by U.S. National Science Foundation grants No. DMR-9634873 and DMR-9981815, and by Florida State University through the Center for Materials Research and Technology and the School of Computational Science and Information Technology. 
* $\quad$ Electronic address: fujisaka@acs.i.kyoto-u.ac.jp

$\dagger \quad$ Electronic address: tutu@acs.i.kyoto-u.ac.jp

¥ Electronic address: rikvold@csit.fsu.edu

[1] E. Warburg, Annalen der Physik und Chemie (Neue Folge) 13, 141 (1881).

[2] J. A. Ewing, Proc. R. Soc. London 34, 39 (1882).

[3] Q. Jiang, H.-N. Yang, and G.-C. Wang, Phys. Rev. B 52, 14911 (1995).

[4] Q. Jiang, H.-N. Yang, and G.-C. Wang, J. Appl. Phys. 79, 5122 (1996).

[5] Y. Ishibashi and Y. Takagi, J. Phys. Soc. Jpn. 31, 506 (1971).

[6] S. Hashimoto, H. Orihara, and Y. Ishibashi, J. Phys. Soc. Jpn. 63, 1601 (1994).

[7] P. D. Beale, Integrated Ferroelectrics 4, 107 (1994).

[8] V. N. Smelyanskiy et al., J. Chem. Phys. 110, 11488 (1999).

[9] S. J. Mitchell, G. Brown, and P. A. Rikvold, J. Electroanal. Chem. (in press). E-print: cond-mat/0005249.

[10] S. J. Mitchell, G. Brown, and P. A. Rikvold, Surf. Sci. (submitted). E-print: cond-mat/0007079.

[11] A. Chen and M. Caffrey, J. Phys. Chem. 100, 5608 (1996).

[12] M. Acharyya, Phys. Rev. E 56, 2407 (1997).

[13] S. W. Sides, P. A. Rikvold, and M. A. Novotny, Phys. Rev. Lett. 81, 834 (1998).

[14] S. W. Sides, P. A. Rikvold, and M. A. Novotny, Phys. Rev. E 59, 2710 (1999).

[15] P. A. Rikvold et al., in Computer Simulation Studies in Condensed Matter Physics XIII, edited by D. P. Landau, S. P. Lewis, and H.-B. Schüttler (Springer, Berlin, 2000), pp. 105-119.

[16] G. Korniss, C. J. White, P. A. Rikvold, and M. A. Novotny, Phys. Rev. E (submitted). E-print: cond-mat/0008155.

[17] G. Korniss, P. A. Rikvold, and M. A. Novotny, Phys. Rev. E (in preparation).

[18] T. Tomé and M. J. de Oliveira, Phys. Rev. A 41, 4251 (1990).

[19] J. F. F. Mendes and E. J. S. Lage, J. Stat. Phys. 64, 653 (1991).

[20] W. S. Lo and R. A. Pelcovits, Phys. Rev. A 42, 7471 (1990).

[21] M. Acharyya and B. Chakrabarti, Phys. Rev. B 52, 6550 (1995).

[22] M. Acharyya, Phys. Rev. E 56, 1234 (1997).

[23] M. Acharyya, Phys. Rev. E 58, 179 (1998).

[24] M. Acharyya, Phys. Rev. E 59, 218 (1999).

[25] G. M. Buendía and E. Machado, Phys. Rev. B 61, 14686 (2000).

[26] M. Zimmer, Phys. Rev. E 47, 3950 (1993).

[27] G. M. Buendía and E. Machado, Phys. Rev. E 58, 1260 (1998).

[28] M. Acharyya and B. K. Chakrabarti, in Annual Reviews of Computational Physics I, edited by D. Stauffer (World Scientific, Singapore, 1994), p. 107.

[29] B. Chakrabarti and M. Acharyya, Rev. Mod. Phys. 71, 847 (1999).

[30] G. Grinstein, C. Jayaprakash, and Y. He, Phys. Rev. Lett. 55, 2527 (1985).

[31] V. A. Yakubovich and V. M. Starzhinskii, Linear Differential Equations with Periodic Coefficients, Vols. 1 and 2 (Keter Publishing House, Jerusalem, 1975); A. H. Nayfeh and D. T. Mook, Nonlinear Oscillations (Wiley, New York, 1979).

[32] See, e.g., N. Goldenfeld, Lectures on Phase Transitions and the Renormalization Group (Addison-Wesley, Reading, MA, 1992).

[33] A. C. Brańka and D. M. Heyes, Phys. Rev. E 58, 2611 (1998).

[34] N. G. van Kampen, Stochastic Processes in Physics and Chemistry, Revised and enlarged edition (North-Holland, Amsterdam, 1992).

[35] H. Risken, The Fokker-Planck Equation, Method of Solution and Applications (Springer, Berlin, 1996).

[36] G. Porod, in Small Angle X-ray Scattering, edited by O. Glatter and I. Kratky (Academic, New York, 1983); A. Guinier and G. Fournet, Small-Angle Scattering of X-rays (Wiley, New York, 1955). 

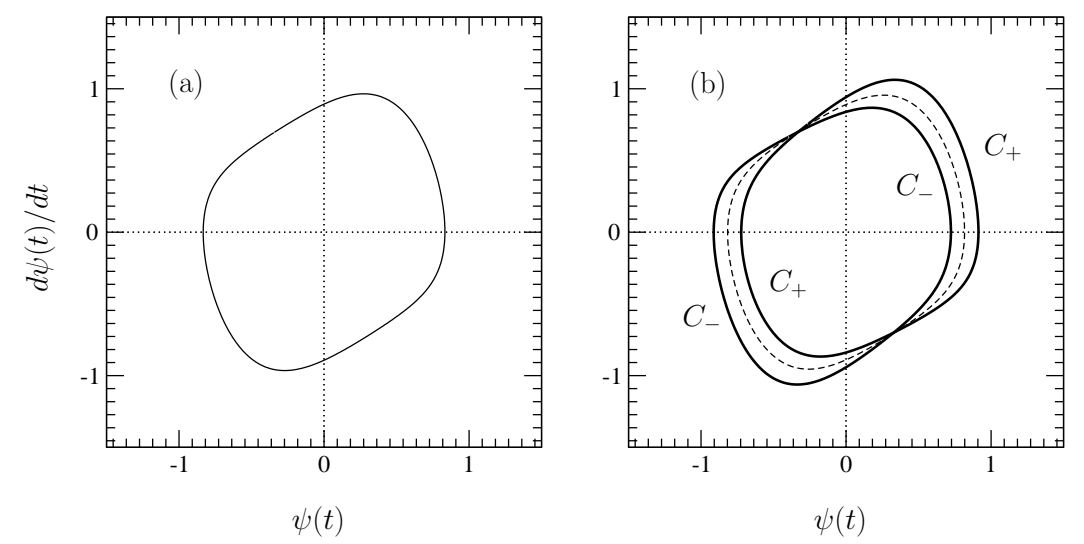

Fig. 1

FIG. 1. Limit-cycle attractors for parameter values (a) $h=1.0, \Omega=1.08$ and (b) $h=1.0, \Omega=1.1$. The phase points move clockwise. In (a) $\left(\Omega<\Omega_{c} \approx 1.095\right)$, there stably exists only one limit cycle, which is symmetric in the sense that Eq. $(6)$ is satisfied. In (b) $\left(\Omega>\Omega_{c}\right)$, the symmetric limit cycle denoted by the dashed curve is unstable, and there appear two stable non-symmetric limit cycles, $\mathrm{C}_{+}$and $\mathrm{C}_{-}$.
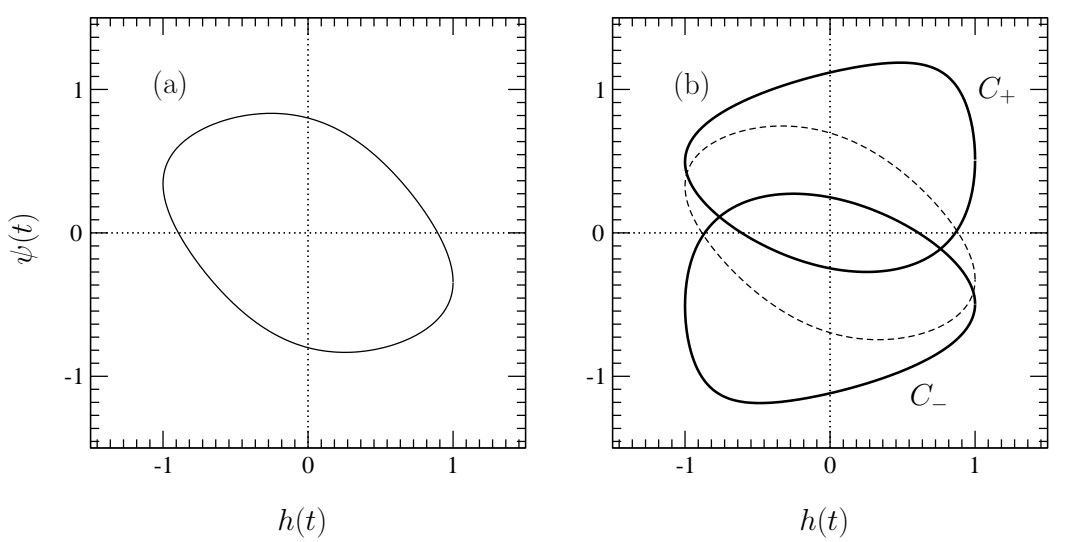

Fig. 2

FIG. 2. Hysteresis loops of limit-cycle attractors, i.e., $\psi(t)$ vs $h(t)=h \cos (\Omega t)$ for (a) $h=1.0, \Omega=1.08$ and (b) $h=1.0$, $\Omega=1.2$. The phase points move counterclockwise. In (a) $\left(\Omega<\Omega_{c} \approx 1.095\right)$, there is only one stable symmetric limit cycle. In (b) $\left(\Omega>\Omega_{c}\right)$, the symmetric limit cycle (dashed curve) is unstable, and there exist two stable non-symmetric limit cycles, $\mathrm{C}_{+}$ and $\mathrm{C}_{-}$. 


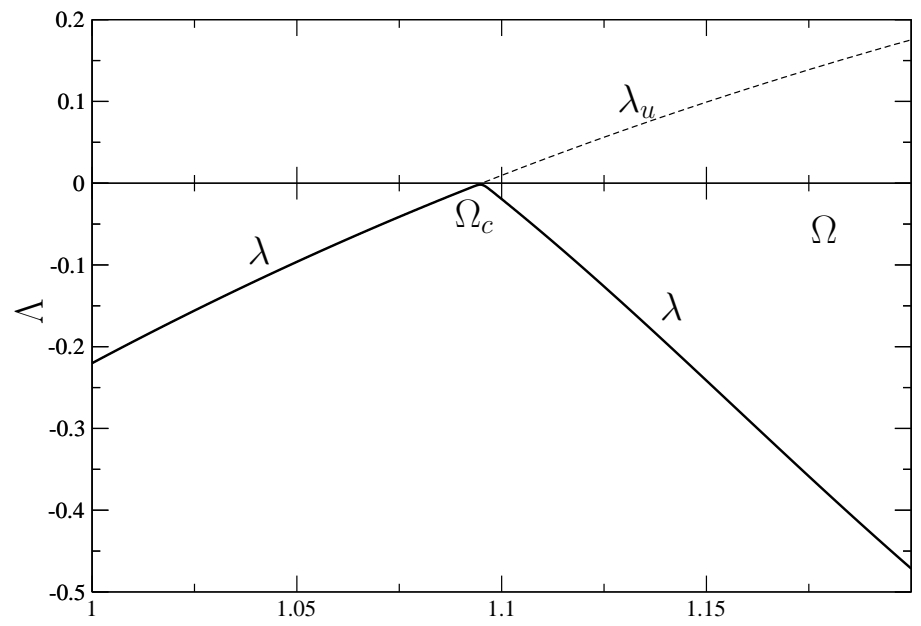

Fig.3

FIG. 3. The Floquet exponent $\Lambda$, shown vs $\Omega$ for $h=1.0$. For $\Omega<\Omega_{c}$, the limit cycle is symmetric and stable. For $\Omega>\Omega_{c}$, the dashed line is the Floquet exponent for the unstable symmetric limit cycle, and the solid line is the exponent for the stable symmetry-breaking limit cycle. For details on the calculation of Floquet exponents, see the text.

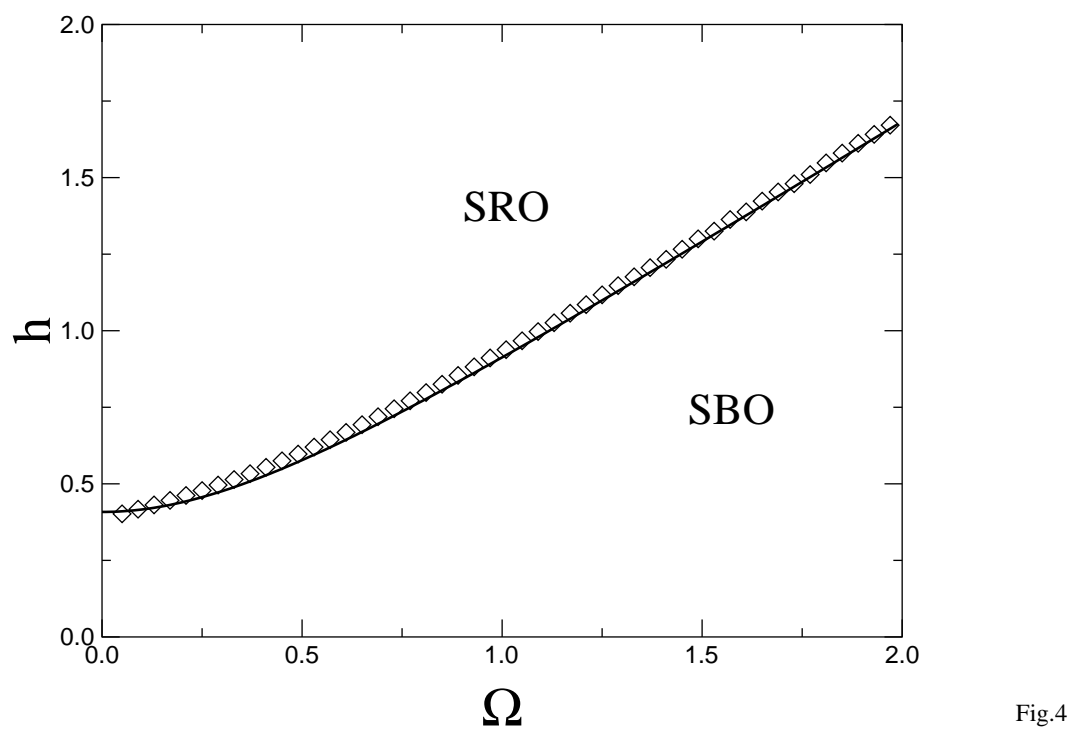

FIG. 4. The bifurcation curve separating the symmetry-restoring oscillation (SRO) and the symmetry-breaking oscillation (SBO). The numerically obtained points are represented as data points, and the approximate theoretical result (21) as a solid curve. 


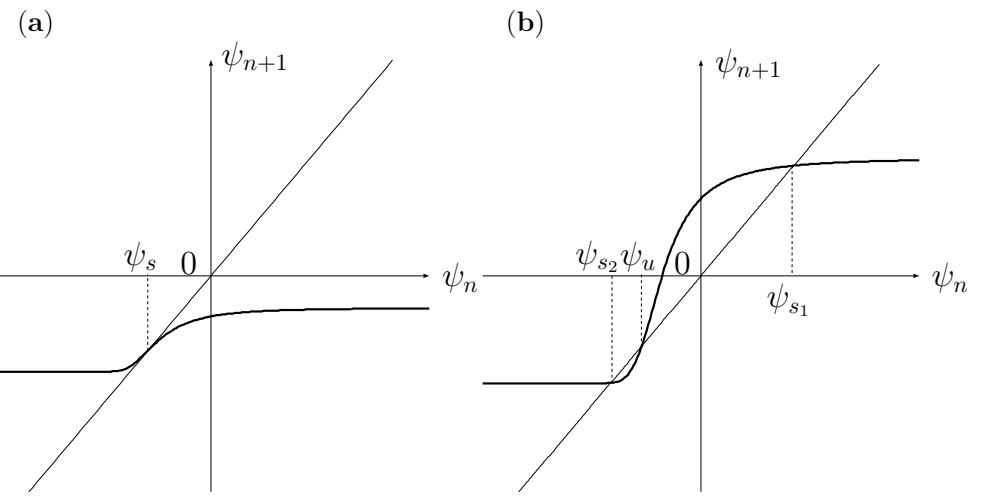

Fig. 5

FIG. 5. Stroboscopic maps $\psi_{n} \equiv \psi(n \cdot 2 \pi / \Omega)$ for (a) $\Omega=1.08\left(<\Omega_{c}\right)$ and (b) $\Omega=1.2\left(>\Omega_{c}\right)$ with $h=1.0$. For $\Omega>\Omega_{c}$, there appear two stable, non-symmetric limit cycles.

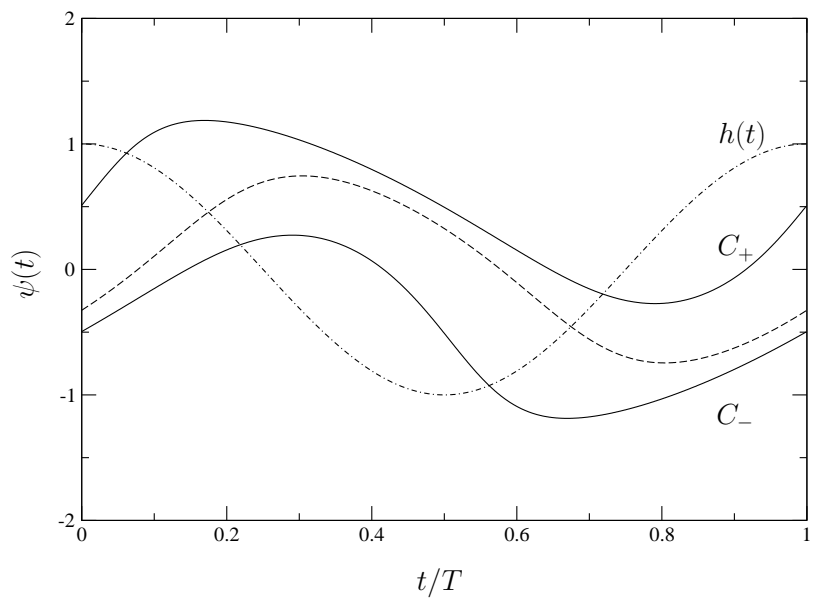

Fig.6

FIG. 6. Temporal evolutions of the stable orbits, $\mathrm{C}_{+}$and $\mathrm{C}_{-}$(solid curves), and the unstable orbit (dashed curve) in the SBO regime for $h=1.0\left(<\Omega_{c}\right)$ and $\Omega=1.2\left(>\Omega_{c}\right)$. The dot-dashed curve represents $h(t)$. 


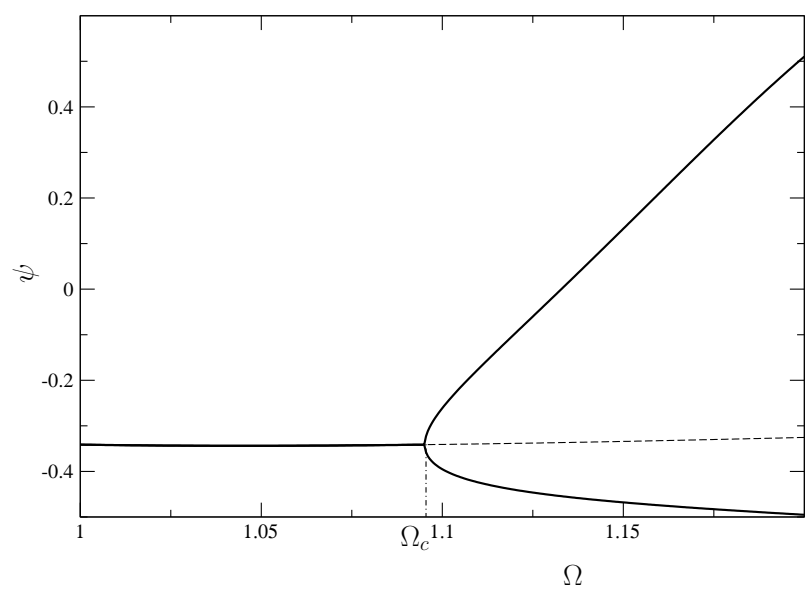

Fig.7

FIG. 7. Bifurcation diagram of $\psi_{n}(\equiv \psi(n \cdot 2 \pi / \Omega))$, i.e., the fixed points of the map 13 for $h=1.0$. For $\Omega<\Omega_{c} \approx 1.095$, there is one unique fixed point. For $\Omega>\Omega_{c}$, there exist one unstable fixed point (dashed line) and two stable fixed points (solid curves).
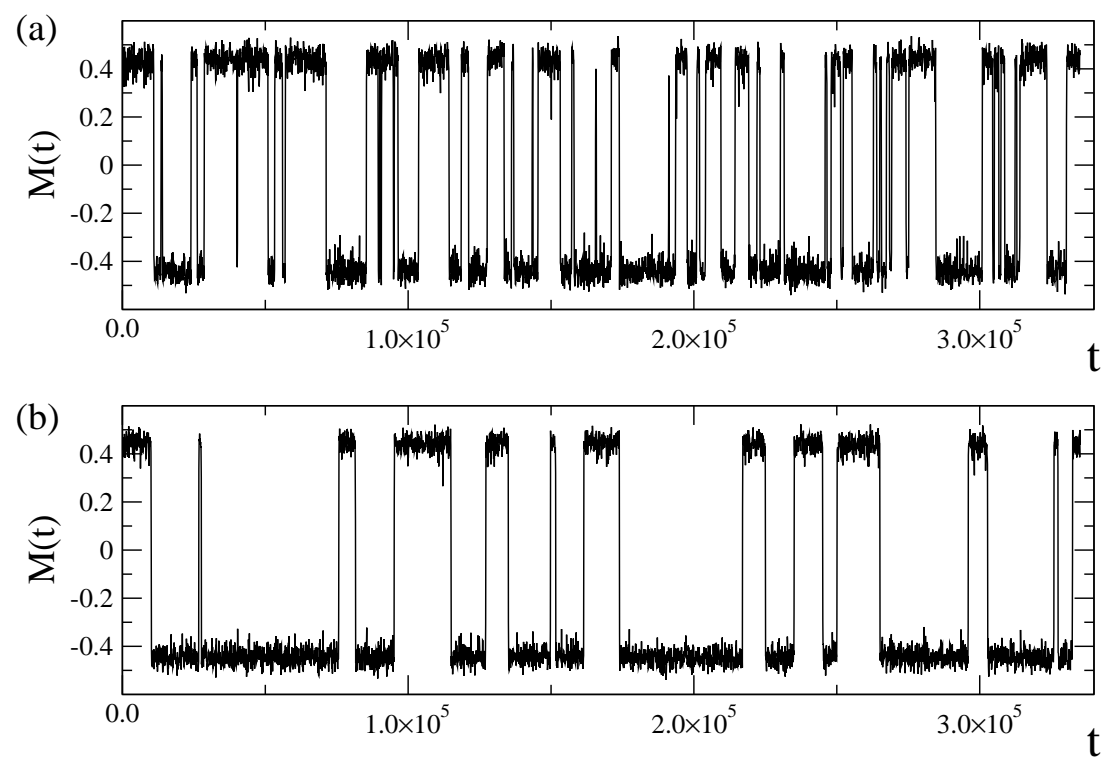

Fig.8

FIG. 8. Switching phenomenon generated by Eq. (8) in a one-dimensional system for $\Omega$ slightly larger than the mean-field value of $\Omega_{c}$. Here $M(t)=L^{-1} \int \overline{\psi(x, t)}^{(16 T)} d x$ is the total magnetization, where $L$ is the system size and $\overline{\psi(x, t)}^{(m T)}=(m T)^{-1} \int_{t}^{t+m T} \psi(x, s) d s$. Parameters are $h=1.0, \Omega=1.2,\left(\Omega_{c} \approx 1.095\right)$ and $\Gamma=0.005$. System sizes are (a) $L=16 \Delta x$, (b) $L=20 \Delta x$, where $\Delta x=0.5$ is the lattice spacing. The time increment is chosen as $\Delta t=T / 1024=0.005113$. Numerical simulations were carried out for $L / \Delta x=64,80,96,112,128,144,160,176$, and 192 . The average time between switching events was observed to increase monotonically with $L$. 

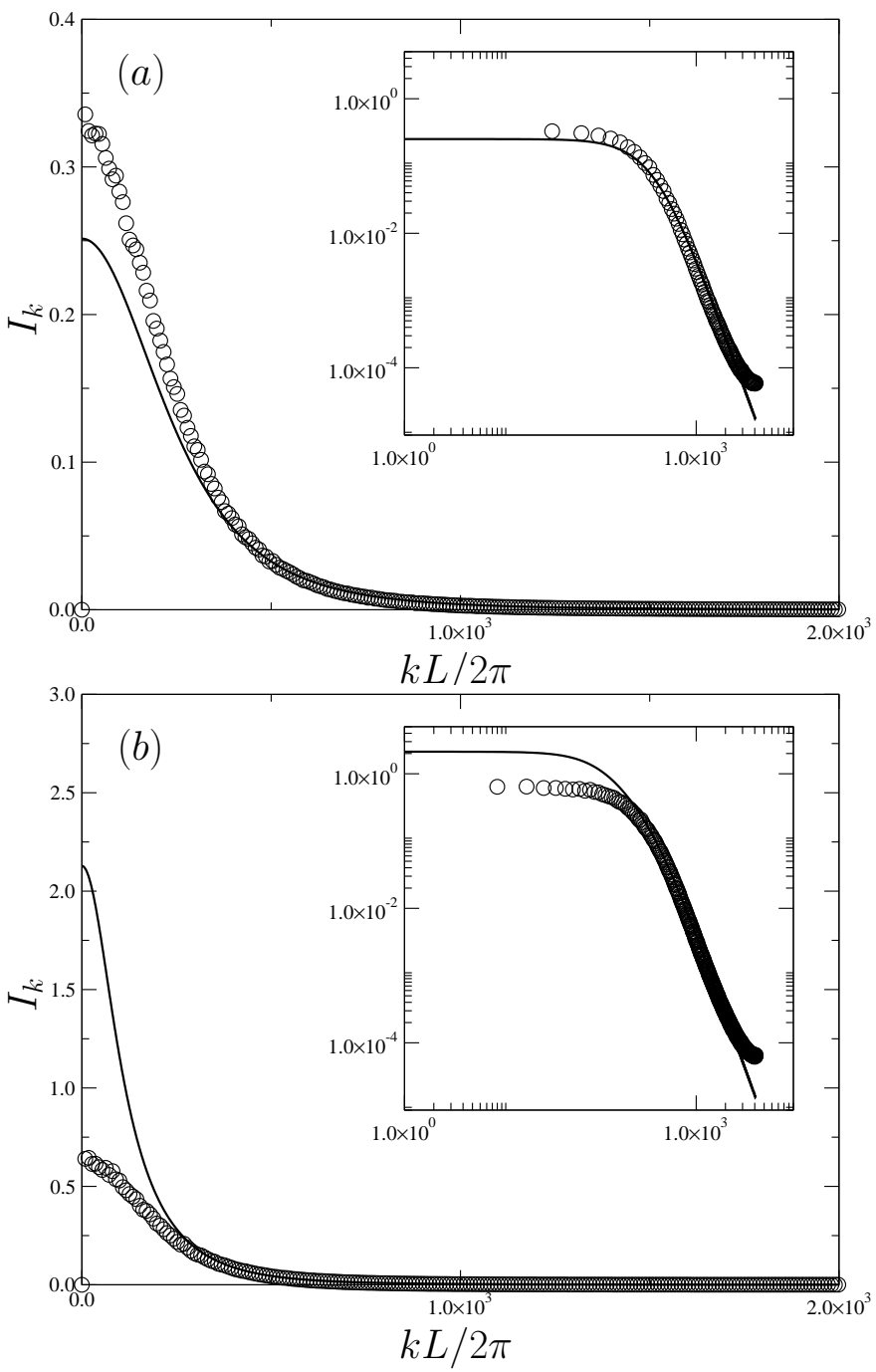

Fig. 9

FIG. 9. The structure functions for the Fourier components of $\overline{\psi(\mathbf{r}, t)}$ in a one-dimensional system with thermal noise in the SRO regime. The numerically obtained results are shown as $\circ$, while the theoretical result of the linearized model (59) is shown as a solid curve. The insets are log-log plots. The parameters are (a) system size $L=8192 \times \Delta x$ with lattice spacing $\Delta x=0.5$, $h=1.0, \Omega=1.0,(\lambda=-0.220)$, and $\Gamma=0.05$, and (b) $L, \Delta x, h$, and $\Gamma$ the same as in (a), while $\Omega=1.08,(\lambda=-0.0307)$. 